\title{
POPULATION STUDIES IN PREDOMINANTLY SELF-POLLINATED SPECIES
}

\section{ANALYSIS OF DIFFERENTIAL AND RANDOM VIABILITIES IN MIXTURES OF COMPETING PURE LINES}

\author{
P. L. WORKMAN * \\ National Institutes of Health, Bethesda, Maryland \\ and \\ R. W. ALLARD \\ Department of Agronomy, University of California, Davis
}

\section{INTRODUCTION}

Received $18 . x .63$

Competition between self-pollinated plants can be studied in experimental populations composed of mixtures of pure lines (e.g. Suneson, I949). The essential features of such studies are: (I) a mixture consisting of known proportions of two or more lines is seeded in a plot, grown to maturity and the seed harvested in mass; (2) a random sample of seeds is drawn from the harvest to establish another plot in the next generation, and so on, for a number of generations; (3) progressive changes in the composition of the mixture are determined by an annual census. Changes in the composition of the mixture can result from the directed process of selection (differential viabilities), sampling variation due to the finite size of the census population, and from random fluctuations of the viabilities. We shall consider in this paper a method for estimating the relative viabilities of the competing lines which takes into account the relevant directed and random processes.

\section{THEORY}

In populations composed of mixtures of say $k$ pure lines in which there may be differential and random viabilities, the proportion $\mathrm{P}_{j}$ of the $j$ th pure line (genotype) in generation $(t+\mathrm{I})$ is given recursively by

$$
\mathrm{P}_{j}(t+\mathrm{I})=x_{j} \mathrm{P}_{j}(t) / \sum_{j=1}^{k} x_{j} \mathrm{P}_{j}(t)
$$

where the $x_{j}$ are random viabilities.

In such a population, for any two lines, say $\mathrm{P}_{i}$ and $\mathrm{P}_{j}$, we see from (I) that

or

$$
\frac{\mathrm{P}_{i}(t+\mathrm{I})}{\mathrm{P}_{j}(t+\mathrm{I})}=\frac{x_{i} \mathrm{P}_{i}(t)}{x_{j} \mathrm{P}_{j}(t)}
$$

$$
\log \left[\frac{\mathrm{P}_{i}(t+\mathrm{I})}{\mathrm{P}_{j}(t+\mathrm{I})}\right]=\log \left(\frac{x_{i}}{x_{j}}\right)+\log \left[\frac{\mathrm{P}_{i}(t)}{\mathrm{P}_{j}(t)}\right] .
$$

\footnotetext{
* Present Address : Department of Genetics, University of Cambridge.
} 
If we let $Z_{i / j}(t+I)=\log \left[\mathrm{P}_{i}(t) / \mathrm{P}_{j}(t)\right]$ so that

then,

$$
\mathrm{Z}_{i / j}(t+\mathrm{I})=\log \left(x_{i} / x_{j}\right)+\mathrm{Z}_{i / j}(t),
$$

$$
\mathrm{Z}_{i / j}(\mathrm{~T})=\mathrm{Z}_{i / j}(\mathrm{I})+\sum_{i=1}^{\mathrm{T}-1} \xi_{i / j}(t),(\mathrm{T} \geq 2)
$$

for $t=\mathrm{I}, 2, \ldots, \mathrm{T}$, where $\xi_{i / j}(t)$ is the value of $\log \left(x_{i} / x_{j}\right)$ realised in the $t$ th generation, computed from (3). Equation (5) is the exact solution based on the discrete model and shows that if $\log \left(x_{i} / x_{j}\right)$ distributes normally with mean $M_{i / j}$ and variance $V_{i / j}$, then $Z_{i / j}(T)$ also distributes normally with mean $Z_{i / j}(\mathrm{I})+(\mathrm{T}-\mathrm{I}) \mathbf{M}_{i / j}$ and variance $(\mathrm{T}-2) \mathrm{V}_{i / j}$. For the bivariate case, $\mathrm{k}=2$, the relation $\mathrm{Z}=\log$ $\mathrm{P} /(\mathrm{I}-\mathrm{P})$ was shown to be normal by Fisher (1922). By the general regularity conditions of the central limit theorem, $\Sigma \xi_{i / j}(t)$ is asymptotically normally distributed and hence so is $Z_{i / j}$ (Gramer, I946). From this we see that $\log \left(x_{i} / x_{j}\right)$, which equals the difference of two approximately normal variables, is also approximately normal.

The estimation of the relative viabilities proceeds as follows. Equation (3) shows that the expected value of $\log \left(x_{i} / x_{j}\right)$ can be estimated for pairs of successive generations by the difference of the logarithmic genotypic ratios without any assumptions about the distributions of the $\mathrm{P}_{j}$ or the $x_{j}$. Since we are estimating the relative viabilities, we can let $x_{k}=\mathrm{I}$, for some $k$, and compute the values of $\log \left(x_{j} / x_{k}\right)=\log \left(y_{j}\right)$ for all $j \neq k$. Thus the viabilities of each line $j$ relative to some base line $k$ are now given by

$$
y_{1}, y_{2}, \ldots, \mathrm{I} \text {, or } x_{1} / x_{k}, x_{2} / x_{k}, \ldots, x_{k-1} / x_{k}, x_{k} / x_{k}=\mathrm{I} \text {. }
$$

Then, for all lines $\mathrm{P}_{j}(j \neq k)$ and for every pair of consecutive generations, say the $t$ th and $(t+\mathrm{I})$ st, the values of $\log \left(y_{j}\right)$ are computed from

$$
u_{j}(t)=\log \left[y_{j}(t)\right]=\log \frac{\mathrm{P}_{j}(t+\mathrm{I})}{\mathrm{P}_{k}(t+\mathrm{I})}-\log \frac{\mathrm{P}_{j}(t)}{\mathrm{P}_{k}(t)} .
$$

The $t$ th estimate of the log of the viability ratio, $u_{j}(t)$, will deviate from the mean viability $\mu_{j}$, by a factor $v_{j}(t)$ due to fluctuations about the mean viability, and by a factor $e_{j}(t)$, due to sampling error resulting from estimating the $\mathrm{P}_{j}(t)$ from a finite census population. That is,

$$
u_{j}(t)=\mu_{j}+v_{j}(t)+e_{j}(t) .
$$

We shall assume that $v_{j}(t)=\mathrm{N}\left(0, \sigma_{v j}^{2}\right), e_{j}(t)=\mathrm{N}\left(\mathrm{o}, \sigma_{e_{j}}^{2}\right)$ and that $v_{j}$ is independent of $e_{j}$. Then, $u_{j}(t)=\mathbf{N}\left(\mu_{j}, \sigma_{u j}^{2}\right)$, where $\sigma_{u j}^{2}=\sigma_{v_{j}}^{2}+\sigma_{\theta_{j}}^{2}$ for all $t$. 
For each estimate of $\mu_{j}$ there will be a corresponding estimate of $\sigma_{e,}^{2}$ given by

$$
s_{e j}^{2}(t) \simeq \frac{\mathrm{I}}{\mathrm{N}(t)}\left\{\frac{\mathrm{I}}{\mathrm{P}_{j}(t)}+\frac{\mathrm{I}}{\mathrm{P}_{k}(t)}\right\}+\frac{\mathrm{I}}{\mathrm{N}(t+\mathrm{I})}\left\{\frac{\mathrm{I}}{\mathrm{P}_{j}(t+\mathrm{I})}+\frac{\mathrm{I}}{\mathrm{P}_{k}(t+\mathrm{I})}\right\}
$$

where $\mathrm{N}(t)$ is the size of the census population in the $t$ th generation. Formula (8) is based on the result that if $f_{1}$ and $f_{2}$ are functions of single random variables, $x_{1}$ and $x_{2}$, then for large samples, i.e., $\mathrm{N}(t)$ large, approximately,

$\operatorname{Var} f_{1}=\left[\operatorname{Var} x_{i}\right]\left(\frac{d f_{i}}{d x_{i}}\right)^{2}$, and $\operatorname{cov}\left(f_{1}, f_{2}\right)=\left[\operatorname{cov}\left(x_{1}, x_{2}\right)\right]\left(\frac{d f_{1}}{d x_{1}}\right)\left(\frac{d f_{2}}{d x_{2}}\right)$

(Kendall and Stuart, 1958). In particular,

$$
\operatorname{Var}\left[u_{j}(t)\right]=\operatorname{Var} \log \frac{\mathrm{P}_{j}(t)}{\mathrm{P}_{k}(t)}+\operatorname{Var} \log \frac{\mathrm{P}_{j}(t+\mathrm{I})}{\mathrm{P}_{k}(t+\mathrm{I})} .
$$

Since $\operatorname{Var}\left[\log \left(\mathrm{P}_{j} / \mathrm{P}_{k}\right)\right]=\operatorname{Var}\left(\log \mathrm{P}_{j}\right)+\operatorname{Var}\left(\log \mathrm{P}_{k}\right)-2 \operatorname{cov}\left(\log \mathrm{P}_{j}\right.$, $\left.\log \mathrm{P}_{k}\right)$, and since $\operatorname{Var}\left(\mathrm{P}_{j}\right)=\mathrm{P}_{j}\left(\mathrm{I}-\mathrm{P}_{j}\right) / \mathrm{N}$ and $\operatorname{cov}\left(\mathrm{P}_{j}, \mathrm{P}_{k}\right)=-\mathrm{P}_{j} \mathrm{P}_{k} / \mathrm{N}$ we find that $\operatorname{Var}\left[\log \left(\mathrm{P}_{j} / \mathrm{P}_{k}\right)\right]=\frac{\mathrm{I}}{\mathrm{N}}\left(\frac{\mathrm{I}}{\mathrm{P}_{j}}+\frac{\mathrm{I}}{\mathrm{P}_{k}}\right)$, and (8) follows directly.

Now, for every pair of consecutive generations, we have estimates, $u_{j}(t)$, of the $\log$ of the viability ratio and corresponding estimates, $s_{e j}^{2}(t)$, of the variance due to sampling error. We require estimates of $\mu_{j}$ (the expected value of $\left.u_{j}(t)\right), \sigma_{u_{j}}^{2}$, and $\sigma_{v j}^{2}$. For samples of infinite size we would have $\sigma_{e j}^{2}=0$ and consequently $\sigma_{u j}^{2}=\sigma_{v j}^{2}$. In this case, the desired estimates could be obtained from the standard formulas

$$
\bar{u}_{j}=\frac{\sum_{t=1}^{\mathrm{T}-1} u_{j}(t)}{\mathrm{T}-\mathrm{I}} ; \mathrm{E}\left(\bar{u}_{j}\right)=\mu_{j}
$$

and

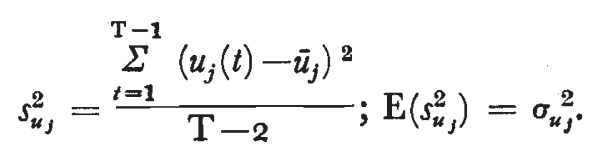

If the census populations are finite, and the $s_{e_{j}}^{2}(t)$ are approximately equal, formulas (9) and (10) can also be applied, and $\sigma_{v_{j}}^{2}$ is estimated by $s_{v_{j}}^{2}=s_{u_{j}}^{2}-\overline{s_{e j}^{2}}(t)$. For these cases, $\frac{\sqrt{\mathrm{T}-\mathrm{I}}\left(\bar{u}_{j}-\mu_{j}\right)}{s_{u_{j}}}$ has "Student's" $t$ distribution and confidence limits for $\mu_{j}$ can be found by standard methods. 
In general, however, the estimates of $s_{e j}^{2}(t)$ will not be equal, and the desired estimates should be obtained as follows. We can write the distribution of the $u_{j}(t)$ as,

$$
\begin{aligned}
f\left(u_{j}(\mathrm{I}), \ldots, u_{j}(\mathrm{~T}-\mathrm{I})\right), \\
\quad=\left\{\frac{\mathrm{I}}{(2 \Gamma)^{n / 2}{ }_{t=1}^{\mathrm{T}-1}\left[\sigma_{v_{j}}^{2}+\sigma_{\epsilon_{j}}^{2}(t)\right]^{1 / 2}}\right\} \exp -\frac{1}{2}\left\{\sum_{t} \frac{\left[u_{j}(t)-\mu\right]^{2}}{\sigma_{v_{j}}^{2}+\sigma_{\epsilon_{j}}^{2}(t)}\right\}
\end{aligned}
$$

for the $(\mathrm{T}-\mathrm{I})$ estimates of $u_{j}(t)$ obtained from data spanning $\mathrm{T}$ consecutive generations. The joint maximum likelihood estimates of $\sigma_{v_{j}}^{2}$ and $\mu_{j}$, namely $\hat{\sigma}_{v_{j}}^{2}$ and $\hat{\mu}_{j}$, are obtained from the simultaneous solution of the equations

and

$$
\hat{\mu}_{j}=\frac{\sum u_{j}(t) /\left[\hat{\sigma}_{v_{j}}^{2}+s_{e j}^{2}(t)\right]}{\sum_{t} \mathrm{I} /\left[\hat{\sigma}_{v_{j}}^{2}+s_{e_{j}}^{2}(t)\right]},
$$

$$
\sum_{t} \frac{\mathrm{I}}{\hat{\sigma}_{v_{j}}^{2}+s_{e_{j}}^{2}(t)}=\Sigma \frac{\left[u_{j}(t)-\hat{\mu}_{j}\right]^{2}}{\left[\hat{\sigma}_{v_{j}}^{2}+s_{e_{j}}^{2}(t)\right]^{2}}
$$

Since the solution must be obtained by iteration, for large $\mathrm{T}$, automatic computers may be required. Equation (12) shows that the maximum likelihood estimate of $\mu_{j}$ is the weighted mean which, for the case where $s_{e j}^{2}(t)$ is either zero or approximately constant, reduces to $(9)$. When the proportions $\mathrm{P}_{j}(t)$ are small, formula (8) shows that $s_{e j}^{2}(t)$ can be quite large and therefore an inefficient estimate may result if this weighted mean is not used.

Since $u_{j}(t)$ is a normal variate, and since $\left(\hat{\mu}_{j}-\mu_{j}\right) / \hat{\sigma}_{\rho_{j}}$ does not follow "Students" $t$ distribution, exact confidence limits can not be obtained, but say, 95 per cent. confidence limits on $\mu_{j}$ are given approximately as $\hat{\mu}_{j} \pm \mathrm{I} \cdot 96 \hat{\sigma}_{\hat{\beta}_{j}}$, for large $\mathrm{T}$, where $\hat{\sigma}_{\hat{\beta}_{j}}^{2}$ is given by (Finney, 1952)

$$
\hat{\sigma}_{a_{j}}^{2} \cong \sum_{t} \frac{\mathrm{I}}{\hat{\sigma}_{v_{j}}^{2}+s_{e_{j}}^{2}(t)} .
$$

These results enable one to decide whether the observed changes in genotypic proportions could be due to sampling variation alone or to actual differences in the viabilities.

The mean and variance of the $y_{j}$ can be obtained from the following formulas (Parzen, 1960). If $\log y_{j}=u_{j}$ is normally distributed with mean $\mathbf{M}_{j}$ and variance $\mathrm{V}_{j}\left(\right.$ i.e. $\log y_{j}=\mathrm{N}\left(\mathrm{M}_{j}, \mathrm{~V}_{j}\right)$ then the mean and variance of $y_{j}$ are given by

and

$$
\mathrm{E}\left(y_{j}\right)=\exp \left(\mathrm{M}_{j}+\frac{1}{2} \mathrm{~V}_{j}\right)
$$

$$
\operatorname{Var}\left(y_{j}\right)=\exp \left(2 \mathrm{M}_{j}+2 \mathrm{~V}_{j}\right)-\exp \left(2 \mathrm{M}_{j}+\mathrm{V}_{j}\right) \text {, }
$$

where the estimates of $\mathbf{M}_{j}$ and $\mathrm{V}_{j}$ are $\bar{u}_{j}$ and $s_{u_{j}}^{2}$, respectively. 
It is possible that the relative viabilities in successive generations are not independent random variables but reflect correlations due to cyclic changes or some other factors causing dependence. In addition, the successive estimates can be independent but have a mean value which is shifting. Under such conditions the estimate of the variance, $s_{v_{j}}^{2}$, obtained from (12) and (13) may be a considerable overestimate of the variance of $\mu_{j}$.

An alternative estimate of the variance can be obtained by calculating the mean square successive differences of the $u_{j}(t)$, and then calculating $q_{j}^{2}$ where $\mathrm{E}\left(q_{j}^{2}\right)=\mathrm{V}_{j}$ and $q_{j}^{2}$ is given by (Hald, I952)

$$
q_{j}^{2}=\frac{\mathrm{I}}{2(\mathrm{~T}-2)} \stackrel{\mathrm{T}}{\mathrm{T}}-2_{t=1}^{2}\left[u_{j}(t+\mathrm{I})-u_{j}(t)\right]^{2} .
$$

$q_{j}^{2}$ can be compared with the total variance, $s_{u j}^{2}$, due to both fluctuations and sampling error, given by (Io). The ratio $r=q_{j}^{2} / s_{u_{j}}^{2}$ provides a significance test for the hypothesis that a changing mean has taken place as opposed to the hypothesis of independence (see Hald, 1952, for details). If $q_{j}^{2} \cong s_{u j}^{2}$, then dependence or a changing mean is unlikely and the above procedures can be applied to the data. However, if $q_{j}^{2} \ll s_{u_{j}}^{2}$ then dependence or a changing mean can be inferred. In such cases it may be possible to partition the data into groups of consecutive generations with equal mean viabilities and consider each group separately.

\section{APPLICATION \\ (i) The experimental data}

The application of the preceding technique to experimental data will be illustrated by an analysis of the relative viabilities of competing varieties of barley studied by Suneson (1949). In Suneson's experiment, the initial mixture was composed of equal proportions of four barley varieties, Atlas (A); Club Mariout (CM); Hero (H); and Vaughn $(\mathrm{V})$, and a plot of $\mathrm{I} / 50$ acre (containing approximately I 5,000 plants) was grown for 16 consecutive generations (1933-48). The percentage of each variety, in each of the generations, is given in table I, and shows the progressive changes in the composition of the mixture. For the first five generations, the proportions were determined from a census of 500 plants; for the remaining generations, the census was based on 1500 plants.

\section{(ii) Estimation of the relevant statistics}

The first step in the analysis requires the calculation, for each pair of generations $t$ and $(t+\mathrm{I})$, of $(a)$ the viability ratios, $y_{j}=x_{j} / x_{k}$; (b) the logarithms of the viability ratios, $u_{j}=\log _{e} y_{j}$; and (c) the standard errors associated with the $u_{j}$, denoted by $s_{e j}^{2}$. Since the proportion of Atlas is always large, we have chosen Atlas as the base 
TABLE $\mathrm{I} *$

Percentage composition of a mixture of four barley varieties grown from successive seedings

\begin{tabular}{|c|c|c|c|c|}
\hline Variety & $\begin{array}{c}\text { Club } \\
\text { Mariout (CM) }\end{array}$ & Hero $(\mathrm{H})$ & Vaughn (V) & Atlas (A) \\
\hline \multicolumn{5}{|l|}{ Generation $(t)$} \\
\hline $\mathrm{I}$ & 24.7 & $24 \cdot 7$ & $25 \cdot 2$ & 25.4 \\
\hline 2 & $23 \cdot 4$ & $20 \cdot 5$ & 18.0 & $38 \cdot 1$ \\
\hline 3 & 18.6 & I5.9 & $18 \cdot 1$ & 47.4 \\
\hline 4 & $22 \cdot 7$ & 12.5 & 19.9 & $42 \cdot 8$ \\
\hline 5 & $24 \cdot 3$ & $12 \cdot 2$ & $14 \cdot 3$ & $49 \cdot 2$ \\
\hline 6 & $20 \cdot I$ & $9 \cdot 2$ & $16 \cdot 2$ & $54 \cdot 4$ \\
\hline 7 & $27 \cdot 6$ & 13.7 & $1 \mathrm{I} \cdot \mathrm{I}$ & $47 \cdot 7$ \\
\hline 8 & $17 \cdot 3$ & $8 \cdot 3$ & II. 3 & 63.1 \\
\hline 9 & 18.8 & $7 \cdot 7$ & $7 \cdot 5$ & 65.5 \\
\hline 10 & $19 \cdot 4$ & $4 \cdot 6$ & 3.4 & $72 \cdot 5$ \\
\hline in & 23.4 & $4 \cdot 4$ & $5 \cdot 4$ & 66.8 \\
\hline $\begin{array}{l}12 \\
13\end{array}$ & $\begin{array}{r}14.6 \\
6.4\end{array}$ & $\begin{array}{l}3.5 \\
2.8\end{array}$ & $\begin{array}{l}2 \cdot 4 \\
2 \cdot 1\end{array}$ & $\begin{array}{l}74 \cdot 5 \\
88 \cdot 7\end{array}$ \\
\hline 14 & $\begin{array}{l}0.4 \\
7.9\end{array}$ & $\mathrm{I} \cdot 5$ & $\begin{array}{l}1 \\
1 \cdot 3\end{array}$ & $89 \cdot 3$ \\
\hline 15 & $8 \cdot 7$ & $1 \cdot 5$ & 1.8 & 86.2 \\
\hline 16 & $10 \cdot 5$ & 0.7 & $0 \cdot 4$ & $88 \cdot 0$ \\
\hline
\end{tabular}

* Adapted from Suneson (1949). Disparity from roo per cent. total represents other varieties of natural hybrids.

variety (i.e. $y_{\mathrm{A}}(t)=\mathrm{I}$ for all $t$ ), minimising the sampling error given by (8). That is, we compute first the relative viability ratios denoted by $x_{\mathrm{CM}} / x_{\mathrm{A}}, x_{\mathrm{H}} / x_{\mathrm{A}}$ and $x_{\mathrm{V}} / x_{\mathrm{A}}$ (or $y_{\mathrm{CM}}, y_{\mathrm{A}}, y_{\mathrm{V}}$ ) where, from (2),

$$
\frac{x_{j}}{x_{k}}(t)=\frac{\mathrm{P}_{j}(t+\mathrm{I}) \mathrm{P}_{k}(t)}{\mathrm{P}_{j}(t) \mathrm{P}_{k}(t+\mathrm{I})},
$$

and then the corresponding logarithmic values and the associated standard errors $\left(u_{j}\right.$ and $s_{e,}^{2}$, for $\left.j=\mathrm{CM}, \mathrm{H}, \mathrm{V}\right)$. The values of the $u_{j}(t)$ and the corresponding standard errors $s_{e j}^{2}(t)$ are given in table 2.

The means and variances of the $u_{j}(t)$, that is, $\bar{u}_{j}$ and $s_{x_{j}}^{2}$, computed according to (9) and (10), as well as the values of $q_{j}^{2}$, from formulas (I 7), are given in table 3 for each of the three comparisons, CM/A, $\mathrm{H} / \mathrm{A}$ and V/A. Comparisons of the $q_{j}^{2}$ with the corresponding $s_{u_{j}}^{2}$ give little indication of either a changing mean or dependence of successive values of $u_{j}(t)$ and we can assume that the value of $\mu_{j}$ is the same for all generations. The error variances, $s_{e j}^{2}(t)$ (table 2) show considerable heterogeneity and when the proportions of the lines become small, the error variance can be quite large relative to the total variance, $s_{u_{j}}^{2}$. Consequently, for this population, the weighted means provide the appropriate estimate of $\mu_{j}$.

Therefore, the next step in the analysis is the computation of the maximum likelihood estimates of the weighted mean $\mu_{j}$, and the variance due to random fluctuations, $\hat{\sigma}_{\hat{v}_{j}}^{2}$. These estimates were 
obtained according to the iteration method described by Bailey ( 1962 ) using an IBM 7094 computer. The values of $\hat{\sigma}_{\rho_{j}}^{2}$, the estimate of the

TABLE 2

Derived values of $u_{j}(t)$ and $s_{c j}^{2}(t)$

\begin{tabular}{|c|c|c|c|c|c|c|}
\hline \multirow{2}{*}{$\begin{array}{c}\text { Comparison } \\
\begin{array}{c}\text { Generation } \\
t\end{array}\end{array}$} & \multicolumn{2}{|c|}{$\mathrm{CM} / \mathrm{A}$} & \multicolumn{2}{|c|}{$\mathrm{H} / \mathrm{A}$} & \multicolumn{2}{|c|}{$\mathrm{V} / \mathrm{A}$} \\
\hline & $u(t)$ & $s_{\theta}^{2}(t)$ & $u(t)$ & $s_{b}^{2}(t)$ & $u(t)$ & $s_{\theta}^{2}(t)$ \\
\hline $\begin{array}{r}1 \\
2 \\
3 \\
4 \\
5 \\
6 \\
7 \\
8 \\
9 \\
10 \\
11 \\
12 \\
13 \\
14 \\
15\end{array}$ & $\begin{array}{r}-0.4956 \\
-0.4480 \\
0.3013 \\
-0.0713 \\
-0.2902 \\
0.4506 \\
-0.7491 \\
0.0458 \\
-0.0701 \\
0.2693 \\
-0.5809 \\
-0.9991 \\
0.2038 \\
0.1318 \\
0.1674\end{array}$ & $\begin{array}{l}0.0300 \\
0.0288 \\
0.0285 \\
0.0258 \\
0.0168 \\
0.0084 \\
0.0087 \\
0.0095 \\
0.0089 \\
0.0082 \\
0.0093 \\
0.0166 \\
0.0204 \\
0.01176 \\
0.0155\end{array}$ & $\begin{array}{r}-0.6279 \\
-0.4725 \\
-0.1385 \\
-0.1637 \\
-0.3827 \\
0.5317 \\
-0.7830 \\
-0.1124 \\
-0.6167 \\
0.0374 \\
-0.3379 \\
-0.3976 \\
-0.6309 \\
0.0353 \\
-0.7828\end{array}$ & $\begin{array}{l}0.0313 \\
0.0318 \\
0.0375 \\
0.0411 \\
0.0289 \\
0.0147 \\
0.0154 \\
0.0188 \\
0.0251 \\
0.0316 \\
0.0361 \\
0.0445 \\
0.0698 \\
0.0904 \\
0.1412\end{array}$ & $\begin{array}{r}-0.7780 \\
-0.2129 \\
0.1969 \\
-0.4968 \\
0.0243 \\
-0.2445 \\
-0.2640 \\
-0.4472 \\
-0.8927 \\
0.5445 \\
-0.9200 \\
-0.3080 \\
-0.4863 \\
0.3608 \\
-1.5247\end{array}$ & $\begin{array}{l}0.0325 \\
0.0316 \\
0.0300 \\
0.0328 \\
0.0234 \\
0.0128 \\
0.0144 \\
0.0169 \\
0.0304 \\
0.0339 \\
0.0420 \\
0.0612 \\
0.0845 \\
0.0898 \\
0.2052\end{array}$ \\
\hline
\end{tabular}

TABLE 3

Derived values of the statistics relevant to the analysis of the relative viabilities

\begin{tabular}{|c|c|c|c|c|c|c|}
\hline Comparison & $\bar{u}(t)$ & $s_{u}^{2}$ & $q^{2}$ & $\hat{\sigma}_{v}^{2}$ & a & $\hat{\sigma}_{\hat{\rho}}^{2}$ \\
\hline $\begin{array}{l}\mathrm{CM} / \mathrm{A} \\
\mathrm{H} / \mathrm{A} \\
\mathrm{V} / \mathrm{A}\end{array}$ & $\begin{array}{l}-0.142 \\
-0.323 \\
-0.361\end{array}$ & $\begin{array}{l}0.184 \\
0.130 \\
0.286\end{array}$ & $\begin{array}{l}0.208 \\
0.186 \\
0.370\end{array}$ & $\begin{array}{l}0.159 \\
0.100 \\
0 \cdot 165\end{array}$ & $\begin{array}{l}-0.141 \\
-0.307 \\
-0.33^{0}\end{array}$ & $\begin{array}{l}0.012 \\
0.009 \\
0.014\end{array}$ \\
\hline
\end{tabular}

variance of the weighted mean (14), were computed in a similar fashion. The derived values of $\hat{\sigma}_{v}^{2}, \hat{\mu}_{j}$, and $\hat{\sigma}_{\hat{\beta}_{j}}^{2}$ are given in table 3 .

\section{(iii) Discussion}

The means and variances of the relative viabilities, $y_{j}=x_{j} / x_{k}$, can be obtained from formulas (15) and (16) using $\hat{\mu}_{j}$ and $\hat{\sigma}_{v}^{2}$, for $\mathbf{M}_{j}$ and $\mathrm{V}_{j}$. The mean viabilities, $\bar{y}_{\mathrm{CM}}=0.94 \mathrm{I}, \bar{y}_{\mathrm{H}}=0.773$ and $\bar{y}_{\mathrm{V}}=0.78 \mathrm{I}$ can be interpreted as indicating that for every Ioo progeny left by Atlas, on the average, Club Mariout, Hero and Vaughn left only 94, 77 and 78 plants respectively. The values are quite close to the raw estimates of $\bar{y}_{j},(0.939,0.773$ and $0.79 \mathrm{I})$ computed by

$$
\bar{y}_{j}=\sum_{t=1}^{\mathrm{T}-1} y_{j}(t) /(\mathrm{T}-\mathrm{I}) \text {. }
$$


The variances of the $y_{j}$ were $\operatorname{Var}\left(y_{\mathrm{CM}}\right)=0.153, \operatorname{Var}\left(y_{\mathrm{H}}\right)=0.063$ and $\operatorname{Var}\left(y_{\mathrm{v}}\right)=0 \cdot 109$. The distribution of the $y_{j}$ has not been specified, but it is not likely that it is normal or even approximately normal. Consequently, confidence limits on the $\bar{y}_{j}$ cannot be determined and tests of significance must be carried out on the logarithmic relative viability ratios.

The approximate 95 per cent. confidence limits on the $\mu_{j}, \mu_{j} \pm \mathrm{I} \cdot 96 \sigma$, namely, $-0.14 \mathrm{I} \pm 0.212 ;-0.307 \pm 0.188 ;$ and, $-0.330 \pm 0.229$, for $\mu_{\mathrm{CM}}, \mu_{\mathrm{H}}$ and $\mu_{\mathrm{V}}$ respectively, provide tests of significance for comparing the relative viabilities of the competing pure lines. The inclusion of the value $\mu_{j}=0.0$ (that is, $x_{j} / x_{k}=\mathrm{I} \cdot 0$ ) would indicate that the mean competitive abilities of the $j$ th pure lines and Atlas were not significantly different. From this we see that the mean relative viability of Atlas exceeds that of Hero and also Vaughn, but that the mean competitive abilities of Club Mariout and Atlas are not significantly different. This latter result is, at first, quite surprising considering the final proportions of the two lines in the mixture (table I). However, an examination of the $u_{\mathrm{CM}}(t)$ in table 2 suggests one possible explanation for this result. The first 12 estimates of $u_{C M}(t)$ have a mean of 0.219 , with a variance sufficiently small so that the 95 per cent. confidence limits do not include the value 0.0 . Therefore, in these generations, the relative viability of Club Mariout is significantly less (at the 5 per cent. level) than that of Atlas. For the last three generations, however, the proportion of Club Mariout increased and $\vec{u}_{\mathrm{CM}}=0 \cdot 168$ for these estimates. This suggests that the mean of the relative viability, that is, $\mu_{\mathrm{CM}}$, may have changed, and that the overall mean provides a spurious description of the relative competitive abilities. Although the ratio, $q_{\mathrm{CM}}^{2} / s_{u_{\mathrm{CM}}}^{2}$ was not significant, it is possible that the large variance due to random fluctuations permits the detection of only extremely large changes in the mean. In addition, table 2 shows that seven of the fifteen values of $u_{\mathrm{CM}}(t)$ were positive indicating a relative adaptive advantage for Club Mariout over Atlas in those generations, while Hero and Vaughn had positive values of $u_{j}$ in only three and four generations respectively.

As noted previously, the observed fluctuations of the relative viabilities can result both from variation due to sampling error and from real fluctuations in the relative competitive abilities of the pure lines $\left(\sigma_{v_{j}}^{2}\right)$. The values of $\hat{\sigma}_{v_{j}}^{2}$, given in table 3 , show that there is considerable generation-to-generation fluctuation of the viabilities, not due to sampling error, but presumably related to seasonal changes in the environment. For the comparisons $\mathrm{CM} / \mathrm{A}, \mathrm{H} / \mathrm{A}$ and $\mathrm{V} / \mathrm{A}$, the ratio $\hat{\sigma}_{v_{j}}^{2} / s_{u_{j}}^{2}$ (estimated variance due to random environmental fluctuations divided by total observed variance) was $0.87,0.77$ and 0.58 respectively. Since $s_{u}^{2}$ gives an unreliable estimate of the real variation of the $u_{j}$, particularly for lines which become rare in the population in later generations, the ratio $\hat{\sigma}_{v_{j}}^{2} / s_{u_{j}}^{2}$ must be interpreted cautiously. However, taken at face value the above estimates indicate 
that viabilities fluctuate more widely about their mean values than has commonly been supposed and hence may be an important factor in determining genetic change in populations.

\section{SUMMARY}

A method is developed for estimating the relative viabilities of competing pure lines. The method provides estimates of $(a)$ the mean of the relative viabilities, $(b)$ the variance of the viabilities due to sampling error associated with finite sample size, and $(c)$ the variance due to random fluctuations about the mean viability. Analysis of data from a barley population in which changes in the proportions of four pure lines were studied for 16 generations indicated that the mean relative viabilities of the pure lines were strikingly different $(\mathrm{r}, 0.94,0.77,0.78)$ and that fluctuations about the means, attributable to seasonal fluctuations in environment, were large.

Acknowledgments. - This work was supported in part by a National Institutes of Health grant GM 10-476. We wish to thank Dr Seymour Geisser of the National Institutes of Health, Bethesda, Maryland, and Dr Matoo Kimura of the National Institute of Genetics, Mishima-shi, Japan, for their helpful comments and suggestions.

\section{REFERENCES}

Bailey, N. T. J. I96I. The Mathematical Theory of Genetic Linkage. Oxford Univ. Press.

CRAmer, H. 1946. Mathematical Methods of Statistics. Princeton Univ. Press. FINNEY, D. J. 1952. Statistical Method in Biological Assay. Charles Griffen and Co. FISHER, R. A. I922. On the Dominance Ratio. Proc. Roy. Soc., Edin., 42, 32 I-34I. HALD, A. 1952. Statistical Theory with Engineering Applications. John Wiley and Sons.

Kendall, M. G., AND stuart, A. 1958. The Advanced Theory of Statistics. Vol. $I$. Distribution Theory. Charles Griffen and Co.

Parzen, E. 1960. Modern Probability Theory and its Applications. John Wiley and Sons.

suneson, c. A. 1949. Survival of four barley varieties in a mixture. Agron. Jour., $4 I, 459-46$ I. 